\title{
Direct Adaptive Fuzzy Sliding Mode Control with Variable Universe Fuzzy Switching Term for a Class of MIMO Nonlinear Systems
}

\author{
Guo Haigang, ${ }^{1,}{ }^{2}$ Li Hongxing, ${ }^{1}$ Zhao Weijing, ${ }^{1}$ \\ and Song Zhankui ${ }^{1}$ \\ ${ }^{1}$ Faculty of Electronic Information and Electrical Engineering, Dalian University of Technology, \\ Dalian 116024, China \\ ${ }^{2}$ School of Mathematics and Statistics, Henan University of Science and Technology, \\ Luoyang 471039, China
}

Correspondence should be addressed to Guo Haigang, ghgxsygzy@mail.dlut.edu.cn

Received 8 October 2012; Accepted 18 November 2012

Academic Editor: Hamid Reza Karimi

Copyright (c) 2012 Guo Haigang et al. This is an open access article distributed under the Creative Commons Attribution License, which permits unrestricted use, distribution, and reproduction in any medium, provided the original work is properly cited.

\begin{abstract}
Combining adaptive fuzzy sliding mode control with fuzzy or variable universe fuzzy switching technique, this study develops two novel direct adaptive schemes for a class of MIMO nonlinear systems with uncertainties and external disturbances. The proposed control schemes consist of fuzzy equivalent control terms, fuzzy switching control terms (in scheme one) or variable universe fuzzy switching control terms (in scheme two), and compensation control terms. The compensation control terms are used to relax the assumption on fuzzy approximation error. Based on Lyapunov stability theory, the parameters update laws are adaptively tuned online and the global asymptotic stability of the closed-loop system can be guaranteed. The major contribution of this study is to develop a novel framework for designing direct adaptive fuzzy sliding mode control scheme facing model uncertainties and external disturbances. The derived schemes can effectively solve the chattering problem and the equivalent control calculation in that environment. Simulation results performed on a two-link robotic manipulator demonstrate the feasibility of the proposed control schemes.
\end{abstract}

\section{Introduction}

Some nonlinear systems, such as robotic manipulator, inverted pendulum, and electrical machines, not only are often highly coupled and time-varying systems, but also suffer from structured and unstructured uncertainties [1,2]. The control of these systems is an important topic in the field of control. Sliding mode control (SMC) is an effective control scheme to deal with these problems [3-6]. However, this control scheme suffers mainly from two disadvantages. One is the chattering due to discontinuous switching term. The other is 
the difficulty involved in the calculation of the equivalent control [7]. A thorough knowledge of the plant dynamics is required for this purpose. But in the real world, there are many complex industrial processes whose accurate mathematical models are not available or difficult to formulate.

In recent decades, fuzzy control methodology has emerged as a promising way to approach nonlinear control problems since it can incorporate linguistic information from human experts into control strategy [8-13]. Considerable efforts have been done to combine fuzzy system with SMC to overcome the disadvantages of general SMC [1, 14-30]. For example, fuzzy switching technique [15-17] and fuzzy boundary layer technique [18-20] have been developed to eliminate chattering problem. Both the techniques are built on the condition that the equivalent control has already existed. There exist some difficulties for the techniques to obtain a suitable equivalent control if the nominal mathematics model is unknown. In this case, there are generally two kinds of adaptive fuzzy SMC approaches to calculate the equivalent control in the existing literatures: direct $[1,21,22]$ and indirect approaches [23-27]. Direct approach is to use fuzzy system directly to approximate the equivalent control term. Indirect approach is first to utilize fuzzy systems to approximate the unknown system functions, then to design the equivalent control based on these estimates. Both the approaches can effectively deal with the calculation of the equivalent control in the presence of model uncertainties and unknown disturbance. But the chattering problem might be encountered no matter what type of adaptive fuzzy SMC.

Several indirect schemes which combined adaptive fuzzy SMC with fuzzy switching technique have been reported for SISO nonlinear systems in [28-30]. The proposed schemes can simultaneously overcome the two disadvantages of SMC mentioned above despite of model uncertainties and unknown disturbances. Unfortunately, convergence of the tracking error to zero is guaranteed by assuming that the fuzzy approximation error is very small if not equal to zero and square integrable. This, however, is difficult to show for any given plant [31]. Besides, direct approach may be of more interest not only because of its simple design and easy implementation, but also because it does not need to consider any possible controller singularity problem [32]. However, the constraints on control gain present difficulties for the design of direct adaptive control. Therefore, it will be a challenge for the direct control of MIMO nonlinear systems with model uncertainties and unknown disturbances.

Combining adaptive fuzzy SMC with fuzzy or variable universe fuzzy switching technique, this paper proposes two novel direct adaptive control schemes for a class of MIMO nonlinear systems with uncertainties and external disturbances. The difference between them lies in that one scheme employs fuzzy system to estimate the switching control gain and the other uses the variable universe fuzzy system proposed in [33] to do it. Motivated by paper [21], this study relaxes the constraint on the gain matrix and only requires it to be positive definite symmetric besides the inverse of its derivative is bounded by an unknown function. To relax the assumption on fuzzy approximation error [28-30], we append an adaptive compensation term to compensate the effect of fuzzy approximation error [34]. The overall closed-loop systems stability and the online adjustment laws of the updated parameters are built based on Lyapunov stability theory. Lastly, the proposed schemes are utilized to deal with the trajectory tracking problem of robotic manipulators. Simulation results demonstrate that the proposed schemes are effective for a class of MIMO nonlinear systems. The two control schemes cannot only achieve the asymptotical tracking for ideal input signal, but also effectively eliminate the chattering of the general SMC.

The rest of this paper is organized as follows. In Sections 2 and 3, brief statements about the control system and variable universe fuzzy system are provided, respectively. 
Section 4 develops two novel direct adaptive fuzzy SMC schemes. Computer simulation results are illustrated in Section 5. Section 6 concludes this paper.

\section{Problem Statement}

Consider the following MIMO nonlinear system [21, 25]:

$$
\begin{aligned}
& y_{1}^{\left(r_{1}\right)}=f_{1}(x)+\sum_{j=1}^{p} g_{1 j}(x) u_{j}+d_{1}, \\
& \vdots \\
& y_{p}^{\left(r_{p}\right)}=f_{p}(x)+\sum_{j=1}^{p} g_{p j}(x) u_{j}+d_{p},
\end{aligned}
$$

where $x=\left[y_{1}, \ldots, y_{1}^{\left(r_{1}-1\right)}, y_{2}, \ldots, y_{2}^{\left(r_{2}-1\right)}, \ldots, y_{p}, \ldots, y_{p}^{\left(r_{p}-1\right)}\right]^{T}$ is the state vector which is available for measurement and $r=\left[r_{1}, r_{2}, \ldots, r_{p}\right]^{T}, u=\left[u_{1}, u_{2}, \ldots, u_{p}\right]^{T}$ is the control input vector, $y=\left[y_{1}, y_{2}, \ldots, y_{p}\right]^{T}$ is the output vector, $f_{i}(x)(i=1,2, \ldots, p)$ are unknown continuous nonlinear functions, $g_{i j}(x)(i, j=1,2, \ldots, p)$ are smooth unknown nonlinear functions, and $D(t)=\left[d_{1}, d_{2}, \ldots, d_{p}\right]^{T}$ are unknown external disturbances.

Define $y=\left[y_{1}^{\left(r_{1}\right)}, y_{2}^{\left(r_{2}\right)}, \ldots, y_{p}^{\left(r_{p}\right)}\right]^{T}, F(x)=\left[f_{1}(x), f_{2}(x), \ldots, f_{p}(x)\right]^{T}$, and

$$
G(x)=\left[\begin{array}{ccc}
g_{11}(x) & \ldots, & g_{1 p}(x) \\
\vdots & \ldots, & \vdots \\
g_{p 1}(x), & \ldots, & g_{p p}(x)
\end{array}\right]
$$

Then the dynamic system equation (2.1) can be rewritten as

$$
y^{(r)}=F(x)+G(x) u+D(t) .
$$

Define reference trajectory $x_{d}=\left[x_{d 1}, x_{d 2}, \ldots, x_{d p}\right]^{T}$ and the tracking error

$$
\begin{aligned}
e_{1}(t) & =x_{d 1}(t)-y_{1}(t), \\
& \vdots \\
e_{p}(t) & =x_{d p}(t)-y_{p}(t) .
\end{aligned}
$$


According to $[3,25]$, for each subsystem, one can define sliding surface as follows:

$$
\begin{aligned}
s_{1}(t) & =\left(\frac{d}{d t}+\lambda_{1}\right)^{\left(r_{1}-1\right)} e_{1}(t), \quad \lambda_{1}>0, \\
& \vdots \\
s_{p}(t) & =\left(\frac{d}{d t}+\lambda_{p}\right)^{\left(r_{p}-1\right)} e_{p}(t), \quad \lambda_{p}>0 .
\end{aligned}
$$

From (2.5), we can see that $e_{i}^{(j)}(t) \rightarrow 0, j=0,1, \ldots, r_{i}-1, i=1,2, \ldots, p$ as $s_{i}(t) \rightarrow 0$. Then, the control duty can be transferred to design the control law such that $s_{i}(t) \rightarrow 0, i=1,2, \ldots, p$.

The time derivatives of the sliding surface of each subsystem are

$$
\begin{aligned}
\dot{s}_{1}(t) & =e_{1}^{\left(r_{1}\right)}+\sum_{j=1}^{r_{1}-1} C_{r_{1}-1}^{j} e_{1}^{\left(r_{1}-j\right)} \lambda_{1}^{(j)}=v_{1}-f_{1}(x)-\sum_{j=1}^{p} g_{1 j}(x) u_{j}-d_{1}, \\
& \vdots \\
\dot{s}_{p}(t) & =e_{p}^{\left(r_{p}\right)}+\sum_{j=1}^{r_{p}-1} C_{r_{p}-1}^{j} e_{p}^{\left(r_{p}-j\right)} \lambda_{p}^{(j)}=v_{p}-f_{p}(x)-\sum_{j=1}^{p} g_{p j}(x) u_{j}-d_{p},
\end{aligned}
$$

where $C_{n}^{k}=n ! / k !(n-k)$ ! in which $k \leq n$, and

$$
\begin{gathered}
v_{1}=x_{d 1}^{\left(r_{1}\right)}+\sum_{j=1}^{r_{1}-1} C_{r_{1}-1}^{j} e_{1}^{\left(r_{1}-j\right)} \lambda_{1}^{(j)}, \\
\vdots \\
v_{p}=x_{d p}^{\left(r_{p}\right)}+\sum_{j=1}^{r_{p}-1} C_{r_{p}-1}^{j} e_{p}^{\left(r_{p}-j\right)} \lambda_{p}^{(j)} .
\end{gathered}
$$

Let $S=\left[s_{1}, s_{2}, \ldots, s_{p}\right]^{T}$ and $v=\left[v_{1}, v_{2}, \ldots, v_{p}\right]^{T}$. Equation (2.6) can be rewritten as

$$
\dot{S}=v-F(x)-G(x) u-D(t) .
$$

The objective of this paper is to design a control law $u(t)$ such that the output vector $y(t)$ follows asymptotically the desired trajectory $x_{d}(t)$, with all involved signals in the closed-loop system remaining bounded. In the controller design, the following assumptions are useful for steady analysis and proof.

Assumption 2.1. $G(x)$ is a positive definite and symmetrical matrix.

Assumption 2.2. $(1 / 2)\left\|(d x / d t) G^{-1}(x)\right\| \leq \delta(x)$, where $\delta(x)$ is bounded positive continuous function without knowing its bound. 
Assumption 2.3. The ideal trajectory $x_{d i}(t)(i=1,2, \ldots, p)$ are $r_{i}$-order derivable, and $x_{d i}(t)(i=1,2, \ldots, p)$ and their $j$-order derivatives $\left(j=1,2, \ldots, r_{i}-1\right)$ are continuous functions of known boundary.

Remark 2.4 (see [21]). Assumption 2.1 is useful to the stability analysis and stability proof. There are many physical systems, such as robotic systems and electrical machines, which satisfy the positive definiteness and the symmetry. Assumption 2.2 is not restrictive, since we only assume the existence of $\delta(x)$ and not its knowledge. Moreover, there are several physical systems in which the control gain matrix $G(x)$ satisfies the inequality, for example, robotic manipulators, electrical machines, inverted pendulum, and chaotic systems.

\section{Variable Universe Fuzzy System}

Fuzzy control emerged in decades ago is a promising way to solve nonlinear control problems. It has several excellent properties. For example, it does not require the plant model and can effectively incorporate the semantic knowledge of human experts. Since the universal approximation theorem has been put forward in [10], fuzzy system and fuzzy control evolve faster than before. Specifically, in the control area, fuzzy systems are mainly used as a nonlinear function approximation tool.

It should be emphasized that, in this paper, it is assumed that the structure and the membership function parameters of the fuzzy system are properly specified in advance by the designer. This means that the designer decision is needed to determine the structure of the fuzzy system, namely, the pertinent inputs, the number of membership functions for each input, the membership function parameters, and the number of rules.

\subsection{Fuzzy System}

For convenience, we will recall briefly the fuzzy system in the following. Let $X_{i}=$ $\left[-E_{i}, E_{i}\right](i=1,2, \ldots, m)$ be the universe of input variable $z_{i}(i=1,2, \ldots, m)$ and $Y=[-U, U]$ the universe of output variable $u_{o} \cdot \mathfrak{A}_{i}=\left\{A_{i j}\right\}(j=1,2, \ldots, N)$ is defined as a fuzzy partition on $X_{i}$ and $\mathfrak{B}=\left\{B_{j}\right\}(j=1,2, \ldots, N)$ a fuzzy partition on $Y$, where $A_{i j} \in \mathcal{F}\left(X_{i}\right)$ and $B_{j} \in \mathcal{F}(Y)$ are termed as the base, and $a_{i j}$ and $b_{j}$ are the peak points of $A_{i j}$ and $B_{j}$, respectively. $\mathfrak{A}_{i}$ and $\mathfrak{B}$ are regarded as linguistic variables so that a group of fuzzy inference rules are formed as follows:

$$
\text { If } z_{1} \text { is } A_{1 j}, z_{2} \text { is } A_{2 j}, \ldots, z_{m} \text { is } A_{m j} \text {, then } u_{o} \text { is } B_{j}(j=1,2, \ldots, N) \text {, }
$$

where $N$ represents the number of the rules. Singleton fuzzifier, triangle membership function (overlap law is 0.5), product inference engine, and center average defuzzifier are used in this fuzzy system. The derived output of fuzzy system can be written as

$$
\widehat{u}_{o}(z)=\xi(z)^{T} \theta
$$


where $z=\left[z_{1}, z_{2}, \ldots, z_{m}\right]^{T}, \theta=\left[u_{1}, u_{2}, \ldots, u_{N}\right]^{T}$ is a vector grouping all consequent parameters, and $\xi(z)=\left[\xi_{1}(z), \xi_{2}(z), \ldots, \xi_{N}(z)\right]^{T}$ is fuzzy basis function vector defined as

$$
\xi_{j}(z)=\prod_{i=1}^{m} A_{i j}\left(z_{i}\right), \quad j=1,2, \ldots, N
$$

\subsection{Variable Universe Fuzzy System}

Adaptive fuzzy controller based on fixed universe has limited approximation accuracy according to the interpolation mechanism of fuzzy system [35]. Aiming at the problem, Li first presents the variable universe idea [36]. The core idea of variable universe fuzzy control is that the universes contracts following the decrease of error. Contraction of the universe is equivalent to the increase of the control rules. Therefore, control accuracy is improved.

The so-called variable universe means that some universes, for example, $X_{i}$ and $Y$, respectively, can change along with changing of variables $z_{i}$ and $u_{0}$. In this case, the universes are denoted by

$$
\begin{aligned}
& X_{i}\left(z_{i}\right)=\left[-\alpha_{i}\left(z_{i}\right) E_{i}, \alpha_{i}\left(z_{i}\right) E_{i}\right], \\
& Y\left(u_{o}\right)=\left[-\beta\left(u_{o}\right) U, \beta\left(u_{o}\right) U\right],
\end{aligned}
$$

where $\alpha_{i}\left(z_{i}\right)(i=1,2, \ldots, m)$ and $\beta\left(u_{o}\right)$ are, respectively, called contraction-expansion factors of the universes $X_{i}(i=1,2, \ldots, m)$ and $Y$. Being relative to the variable universes, the original universes $X_{i}$ and $Y$ are naturally called initial universes. After the above changes, the output of variable universe fuzzy controller can be written as

$$
\widehat{u}_{o}(\beta, z)=\beta \zeta(z)^{T} \vartheta
$$

where $\vartheta=\left[u_{2}, u_{2}, \ldots, u_{N}\right]^{T}$ and $\zeta(z)=\left[\zeta_{1}(z), \zeta_{2}(z), \ldots, \zeta_{N}(z)\right]^{T}$ represent the parameter vector of inference consequence and the fuzzy base function vector, respectively, in which $\zeta_{j}(z)=\prod_{i=1}^{m} A_{i j}\left(z_{i} / \alpha_{i}\left(z_{i}\right)\right), j=1,2, \ldots, N$.

Remark 3.1. Under the framework of variable universe fuzzy control, the parameter which needs to be online adjusted is a scalar $\beta$ instead of a parameter vector $\theta$ in conventional adaptive fuzzy control scheme. Therefore, variable universe fuzzy control scheme simplifies the design procedure of adaptive fuzzy control. In addition, we hardly need smart expert knowledge in the realm, but need the rough trend of control rules in the design of variable universe fuzzy controller [37]. From this point, variable universe fuzzy control reduces the design difficulty. 


\section{Adaptive Fuzzy SMC Design}

\subsection{Sliding Mode Control}

In this section, we firstly consider that nonlinear function matrices $F(x)$ and $G(x)$ are known. From Assumption 2.1, the matrix $G(x)$ is reversible. Both sides of (2.8) are multiplied by $G_{1}(x)=G^{-1}(x)$, then we obtain that

$$
G_{1}(x) \dot{S}=G_{1}(x) v-F_{1}(x)-u-G_{1}(x) D(t)
$$

where $F_{1}(x)=G_{1}(x) F(x)$. The control law is designed as

$$
u=G_{1}(x) v-F_{1}(x)-G_{1}(x) D(t)+\delta(x) I_{p} S+\eta \operatorname{sign}(S),
$$

where $\operatorname{sign}(S)=\left[\operatorname{sign}\left(s_{1}\right), \operatorname{sign}\left(s_{2}\right), \ldots, \operatorname{sign}\left(s_{p}\right)\right]^{T}$ and $\eta=\operatorname{diag}\left(\eta_{1}, \eta_{2}, \ldots, \eta_{p}\right)$ in which $\eta_{i}>$ $0(i=1,2, \ldots, p)$, where its aim is to meet the sliding condition. Substituting (4.2) into (4.1), we can obtain

$$
G_{1}(x) \dot{S}=-\eta \operatorname{sign}(S)-\delta(x) I_{p} S
$$

We choose a candidate Lyapunov function $V=(1 / 2) S^{T} G_{1}(x) S$. For the matrix $G(x)$ being positive definite and symmetrical, $G_{1}(x)$ is also positive definite and symmetrical and satisfies $\dot{S}^{T} G_{1}(x) S=S^{T} G_{1}(x) \dot{S}$. Combining with Assumption 2.2, we have

$$
\dot{V}=S^{T} G_{1}(x) \dot{S}+\frac{1}{2} S^{T} \dot{G}_{1}(x) S=-S^{T}\left(\eta \operatorname{sign}(S)+\delta(x) I_{p} S\right)+\frac{1}{2} S^{T} \dot{G}_{1}(x) S \leq-\sum_{i=1}^{p} \eta_{i}\left|s_{i}\right| .
$$

Therefore, $\dot{V}$ is negative definite and the control objective can be achieved. However, in engineering practice, nonlinear system matrix $F(x)$ and control gain matrix $G(x)$ are often unknown. Accordingly, the derivative function matrices $F_{1}(x)$ and $G_{1}(x)$ as well as $\delta(x)$ are also unknown. Moreover, external disturbance vector $D(t)$ is also unknown. Therefore, the control law (4.2) cannot be implemented. In the following, we employ fuzzy systems to design the control law.

\subsection{Direct Adaptive Fuzzy SMC with Fuzzy Switching Term (DAFSMC with FSW)}

According to the sliding mode control scheme, the control law $u$ can be decomposed into the equivalent control and the switching control. In the sliding phase, the role of the equivalent control is to force the system dynamics to stay on the sliding surface. In the reaching phase, the switching control is designed to satisfy the sliding mode condition $[3,28,29]$.

Let

$$
u_{e q} \triangleq G_{1}(x) v-F_{1}(x)-G_{1}(x) D(t)+\delta(x) I_{p} S
$$


and $u_{s w} \triangleq \eta \operatorname{sign}(S)$. Equation (4.2) can rewritten as

$$
u=u_{e q}+u_{s w}
$$

It is well known that the fuzzy rules used for reasoning are not easy to extract, especially for multi-input $(m>2)$ fuzzy system. Yet, a prominent merit of adaptive fuzzy system is that it does not need initial fuzzy rules and can generate fuzzy rules online based on Lyapunov stability theory [38].

So, in what follows, adaptive fuzzy systems as (3.2) are chosen to approximate the equivalent control term $u_{e q}$. Therefore, we have the following format:

$$
u_{e q}=\widehat{u}_{e q}\left(\Theta_{e}^{*}, x\right)+\omega=\Xi_{e}^{T}(x) \Theta_{e}^{*}+\omega,
$$

where $\omega=\left[\omega_{1}, \omega_{2}, \ldots, \omega_{p}\right]^{T}$ and $\Xi_{e}(x)=\left[\operatorname{diag}\left(\xi_{e 1}^{T}(x), \xi_{e 2}^{T}(x), \ldots, \xi_{e p}^{T}(x)\right)\right]^{T}$ denotes fuzzy approximate error vector and fuzzy base function matrix, respectively.

Let $\Theta_{e}^{*}=\left[\theta_{e 1}^{* T}, \theta_{e 2}^{* T}, \ldots, \theta_{e p}^{* T}\right]^{T}$ be the optimal parameter vector, where

$$
\theta_{e i}^{*}=\underset{\left\|\theta_{e i}\right\| \leq M_{e i}}{\arg \min }\left\{\sup _{x \in D_{x}}\left|u_{e q i}-\widehat{u}_{e q i}\left(\theta_{e i}, x\right)\right|\right\}
$$

in which $M_{e i}(i=1,2, \ldots, p)$ is design constant restraining parameter vector $\theta_{e i}(i=$ $1,2, \ldots, p)$ and $D_{x}$ is a compact set containing state $x$, and

$$
\widehat{u}_{e q i}\left(\theta_{e i}, x\right)=\theta_{e i}^{T} \xi_{e i}(x)
$$

denotes the estimate of the equivalent control law $u_{\text {eqi }}$. Due to unknown ideal parameter vector $\theta_{e i}^{*}$, we estimate $\theta_{e i}^{*}$ by virtue of $\theta_{e i}$.

Remark 4.1. In this paper, we assume that fuzzy approximation errors $\omega_{i}(i=1,2, \ldots, p)$ are bounded for all $x \in D_{x}$, that is, $\left|\omega_{i}(x)\right| \leq \rho_{i}$, for all $x \in D_{x}$, where $\rho_{i}$ is an unknown constant. The knowledge of $\rho=\operatorname{diag}\left(\rho_{1}, \rho_{2}, \ldots, \rho_{p}\right)$ is only required for analysis purpose.

Given the approximation error $\omega_{i}$ between the equivalent control $u_{\text {eqi }}$ and the used fuzzy system $\widehat{u}_{e q i}$, the switching control term $u_{s w}$ must be modified as $(\eta+\rho) \operatorname{sign}(S)$, that is, $u_{\text {swi }}=\left(\eta_{i}+\rho_{i}\right) \operatorname{sign}\left(s_{i}\right)(i=1,2, \ldots, p)$. The objective of this modification is to meet the sliding mode existing condition. In the previous literatures $[23,26]$, the switching gain matrix $(\eta+\rho)$ has to be determined in advance. This is difficult when the bound of the approximation error is unknown. Improper switching gain easily causes chattering problem which is undesired in practice.

Since the chattering is caused by the switching gain matrix $(\eta+\rho)$ and the discontinuous function $\operatorname{sign}(s)$, let the switching control $u_{s w}=(\eta+\rho) \operatorname{sign}(s)$ be replaced by a gain vector $K=\left[k_{1}, k_{2}, \ldots, k_{p}\right]$ [17]. Motivated by papers [28-30], in the controller design, we employ fuzzy systems in the form of (3.2) to approximate the gain vector $K$.

According to the switching control $u_{s w i}$ in (4.6), we can choose a single-input singleoutput fuzzy system $\widehat{k}_{i}\left(s_{i}\right)$ to approximate the gain $k_{i}$. Here, the fuzzy system $\widehat{k}_{i}$ is applied to 
compensate the system uncertainty to reduce the energy of $s_{i}$ and causes the sliding surface to approach zero. It is obvious that the sign of $k_{i}$ is the same as that of $s_{i}$. When $\left|s_{i}\right|$ is away from zero, $\left|k_{i}\right|$ should be chosen a large value to make the system move quickly to the switching surface. When $\left|s_{i}\right|$ is small, $\left|k_{i}\right|$ should be chosen a small value to avoid overshoot. When $s_{i}$ is zero, $\left|k_{i}\right|$ should be zero. From the above analysis, it is easy to make $s_{i} \dot{s}_{i} \leq 0$ and guarantee the sliding condition. Briefly, the fuzzy rules can be determined as follows:

$$
\text { If } s_{i} \text { is } A_{h} \text {, then } k_{i} \text { is } B_{h}, h=1,2, \ldots, i_{L} \text {, }
$$

where $s_{i}$ and $k_{i}$ are the input and the output variables of the fuzzy system, respectively, and $i_{L}$ represents the number of fuzzy rules. Then the estimated switching gain $\widehat{k}_{i}$ of the $i$ th subsystem can be written as

$$
\widehat{k}_{i}=\xi_{s i}\left(s_{i}\right)^{T} \theta_{s i}
$$

where $\xi_{s i}\left(s_{i}\right)=\left[A_{1}\left(s_{i} / \alpha_{i}\left(s_{i}\right)\right), \ldots, A_{i_{L}}\left(s_{i} / \alpha_{i}\left(s_{i}\right)\right)\right]^{T}$ and $\theta_{s i}=\left[b_{1}, b_{2}, \ldots, b_{i_{L}}\right]^{T}$. Let

$$
\theta_{s i}^{*}=\underset{\left\|\theta_{s i}\right\| \leq M_{s i}}{\arg \min }\left\{\sup _{s_{i} \in D_{s i}}\left|k_{i}-\xi_{s i}\left(s_{i}\right)^{T} \theta_{s i}\right|\right\}
$$

be the optimal parameter vector, where $M_{s i}$ is design constant restraining parameter vector $\theta_{s i}$ and $D_{s i}$ is compact set containing the variable $s_{i}$. The optimal parameter vector $\theta_{s i}^{*}$ is unknown, so $\theta_{s i}^{*}$ is estimated by $\theta_{s i}$.

Further, to cancel the approximation error between the equivalent control $u_{e q i}$ and the used fuzzy system $\widehat{u}_{\text {eqi }}$, we append a compensation control term $u_{c}=\left[u_{c 1}, u_{c 2}, \ldots, u_{c p}\right]^{T}$ [34]. Therefore, the overall control effort can be modified as

$$
u_{i}=\widehat{u}_{e q i}+\widehat{k}_{i}+u_{c i}=\xi_{e i}^{T}(x) \theta_{e i}+\xi_{s i}^{T}\left(s_{i}\right) \theta_{s i}+u_{c i}
$$

or

$$
u=\widehat{u}_{e q}+\widehat{K}+u_{c}=\Xi_{e}(x)^{T} \Theta_{e}+\Xi_{s}^{T}(S) \Theta_{s}+u_{c},
$$

where $\Xi_{s}(S)=\left[\operatorname{diag}\left(\xi_{s 1}^{T}(s 1), \xi_{s 2}^{T}(s 2), \ldots, \xi_{s p}^{T}(s p)\right)\right]^{T}$ and $\Theta_{s}=\left[\theta_{s 1}^{T}, \theta_{s 2}^{T}, \ldots, \theta_{s p}^{T}\right]^{T}$. The compensation control term $u_{c}$ will be designed next.

Substituting (4.14) into (4.1), we obtain

$$
\begin{aligned}
G_{1}(x) \dot{S} & =G_{1}(x) v-F_{1}(x)-\left(\Xi_{e}^{T}(x) \Theta_{e}+\Xi_{s}^{T}(S) \Theta_{s}+u_{c}\right)-G_{1}(x) D(t) \\
& =\omega-u_{c}-\left(\Xi_{e}^{T}(x) \Theta_{e}-\Xi_{e}^{T}(x) \Theta_{e}^{*}\right)-\left(\Xi_{s}^{T}(S) \Theta_{s}-\Xi_{s}^{T}(S) \Theta_{s}^{*}\right)-\Xi_{s}^{T}(S) \Theta_{s}^{*}-\delta(x) I_{p} S .
\end{aligned}
$$


Let $\widetilde{\Theta}_{e}=\Theta_{e}-\Theta_{e}^{*}$ and $\widetilde{\Theta}_{s}=\Theta_{s}-\Theta_{s}^{*}$. Combining the above equation, we have

$$
G_{1}(x) \dot{S}=\omega-u_{c}-\Xi_{e}^{T}(x) \widetilde{\Theta}_{e}-\Xi_{s}^{T}(S) \widetilde{\Theta}_{s}-\Xi_{s}^{T}(S) \Theta_{s}^{*}-\delta(x) I_{p} S .
$$

To derive the adaptive laws of the parameter vectors, let us consider the Lyapunov candidate function

$$
V_{1}=\frac{1}{2} S^{T} G_{1}(x) S+\frac{1}{2 \gamma_{1}} \widetilde{\Theta}_{e}^{T} \widetilde{\Theta}_{e}+\frac{1}{2 \gamma_{2}} \widetilde{\Theta}_{s}^{T} \widetilde{\Theta}_{s}+\frac{1}{2 \gamma} \tilde{\omega}^{T} \tilde{\omega}
$$

where $\gamma_{1}, \gamma_{2}, \gamma$ are positive constants, $\tilde{\omega}=\omega-\widehat{\omega}$ in which the vector $\widehat{\omega}=\left[\widehat{\omega}_{1}, \widehat{\omega}_{2}, \ldots, \widehat{\omega}_{p}\right]^{T}$ denotes the estimate of the unknown approximation error vector $\omega$.

Theorem 4.2. For system (2.1), nonlinear function matrices $F(x), G(x)$, and vector $D(t)$ are unknown, and Assumptions 2.1-2.3 hold. The control law is selected as (4.13) or (4.14) in which $u_{c i}=\widehat{\omega}_{i}$ with adaptation laws (4.18) as follows:

$$
\begin{gathered}
\dot{\theta}_{e i}= \begin{cases}\gamma_{1} s_{i} \xi_{e i}(x), & \left\|\theta_{e i}\right\|<M_{e i} \text { or }\left\|\theta_{e i}\right\|=M_{e i}, \gamma_{1} s_{i} \theta_{e i}^{T} \xi_{e i}(x) \leq 0, \\
\gamma_{1} s_{i} \xi_{e i}(x)-\gamma_{1} s_{i} \frac{\theta_{e i} \theta_{e i}^{T}}{\left\|\theta_{e i}\right\|^{2}} \xi_{e i}(x), & \left\|\theta_{e i}\right\|=M_{e i}, \gamma_{1} s_{i} \theta_{e i}^{T} \xi_{e i}(x)>0,\end{cases} \\
\dot{\theta}_{s i}= \begin{cases}\gamma_{2} s_{i} \xi_{s i}\left(s_{i}\right), & \left\|\theta_{s i}\right\|<M_{s i} \text { or }\left\|\theta_{s i}\right\|=M_{s i}, \gamma_{2} s_{i} \theta_{s i}^{T} \xi_{s i}\left(s_{i}\right) \leq 0, \\
\gamma_{2} s_{i} \xi_{s i}\left(s_{i}\right)-\gamma_{2} s_{i} \frac{\theta_{s i} \theta_{s i}^{T}}{\left\|\theta_{s i}\right\|^{2}} \xi_{s i}\left(s_{i}\right), & \left\|\theta_{s i}\right\|=M_{s i}, \gamma_{2} s_{i} \theta_{s i}^{T} \xi_{s i}\left(s_{i}\right)>0, \\
\dot{\hat{\omega}}_{i}=\gamma s_{i},\end{cases}
\end{gathered}
$$

where $M_{e i}$ and $M_{s i}$ are defined as before. Then one can derive the performance as follows.

(1) The involved signals of the close loop are bounded.

(2) The tracking errors and their derivatives decay to zero asymptotically, in other words, when $t \rightarrow \infty, e_{i}^{(j)} \rightarrow 0, j=0,1, \ldots, r_{i}-1, i=1,2, \ldots, p$. 
Proof. Differentiating (4.17) with respect to $t$ and using (4.16), we have

$$
\begin{aligned}
\dot{V}_{1}= & S^{T} G_{1}(x) \dot{S}+\frac{1}{2} S^{T} \dot{G}_{1}(x) S+\frac{1}{\gamma_{1}} \tilde{\Theta}_{e}^{T} \dot{\tilde{\Theta}}_{e}+\frac{1}{\gamma_{2}} \tilde{\Theta}_{s}^{T} \dot{\tilde{\Theta}}_{s}+\frac{1}{\gamma} \tilde{\omega}^{T} \dot{\tilde{\omega}} \\
= & S^{T}\left(\omega-u_{c}-\Xi_{e}^{T}(x) \tilde{\Theta}_{e}-\Xi_{s}^{T}(S) \tilde{\Theta}_{s}-\Xi_{s}^{T}(S) \Theta_{s}^{*}-\delta(x) I_{p} S\right) \\
& +\frac{1}{2} S^{T} \dot{G}_{1}(x) S+\frac{1}{\gamma_{1}} \tilde{\Theta}_{e}^{T} \dot{\tilde{\Theta}}_{e}+\frac{1}{\gamma_{2}} \tilde{\Theta}_{s}^{T} \dot{\tilde{\Theta}}_{s}+\frac{1}{\gamma} \tilde{\omega}^{T} \dot{\tilde{\omega}} \\
\leq & \sum_{i=1}^{p} s_{i} \omega_{i}-\sum_{i=1}^{p} s_{i} \widehat{\omega}_{i}-\sum_{i=1}^{p} s_{i} \tilde{\theta}_{e i}^{T} \xi_{e i}(x)+\frac{1}{\gamma_{1}} \sum_{i=1}^{p} \tilde{\theta}_{e i}^{T} \dot{\tilde{\theta}}_{e i}+\frac{1}{2} S^{T} \dot{G}_{1}(x) S-\delta(x)\|S\|^{2} \\
& -\sum_{i=1}^{p} s_{i} \tilde{\theta}_{s i}^{T} \xi_{s i}\left(s_{i}\right)+\frac{1}{\gamma_{2}} \sum_{i=1}^{p} \tilde{\theta}_{s i}^{T} \dot{\tilde{\theta}}_{s i}-\sum_{i=1}^{p} \eta_{i}\left|s_{i}\right|-\frac{1}{\gamma} \sum_{i=1}^{p} \tilde{\omega}_{i}^{T} \dot{\hat{\omega}}_{i} \\
\leq & -\frac{1}{\gamma_{1}} \sum_{i=1}^{p} \tilde{\theta}_{e}^{T}\left(\gamma_{1} s_{i} \xi_{e i}(x)-\dot{\theta}_{e}\right)-\frac{1}{\gamma_{2}} \sum_{i=1}^{p} \tilde{\theta}_{s i}^{T}\left(\gamma_{2} s_{i} \xi_{s i}\left(s_{i}\right)-\dot{\tilde{\theta}}_{s i}\right) \\
& +\frac{1}{\gamma} \sum_{i=1}^{p}\left(\gamma s_{i}\left(\omega_{i}-\widehat{\omega}_{i}\right)-\tilde{\omega}_{i}^{T} \dot{\tilde{\omega}}_{i}\right)-\sum_{i=1}^{p} \eta_{i}\left|s_{i}\right| .
\end{aligned}
$$

Considering the same sign between $s_{i}$ and the fuzzy switching term $k_{i}$, the first inequality in the above derivation is easily established by using the inequality $\eta_{i}\left|s_{i}\right| \leq s_{i} \theta_{s i}^{* T} \xi_{s i}(s i) \leq$ $\left(\eta_{i}+\rho_{i}\right)\left|s_{i}\right|$. Noticing (4.18), we have

$$
\dot{V}_{1} \leq-\sum_{i=1}^{p} \eta_{i}\left|s_{i}\right|<0
$$

Consequently, all signals in the system are bounded. Obviously, if $e(0)$ is bounded, then $e(t)$ is also bounded for all $t$. Since the reference trajectory $x_{d}$ is bounded, then the system state $x(t)$ is bounded as well.

To complete the proof and establish asymptotic convergence of the tracking error, we need to prove that $S \rightarrow 0$ as $t \rightarrow \infty$. We rearrange (4.16) as following:

$$
\dot{S}=G(x)\left(\omega-\widehat{\omega}-\Xi_{e}^{T}(x) \tilde{\Theta}_{e}-\Xi_{s}^{T}(S) \tilde{\Theta}_{s}-\Xi_{s}^{T}(S) \Theta_{s}^{*}-\delta(x) I_{p} S\right) .
$$

Since $G(x)$ and $\delta(x)$ are continuous functions in a compact set $D_{x}$, they are bounded. By using the boundness of $S, \dot{\hat{\omega}} \in L_{\infty}$ and in turn $\widehat{\omega} \in L_{\infty}$. Therefore, $\dot{S} \in L_{\infty}$ holds. Using Barbalat's lemma, $S \rightarrow 0(t \rightarrow \infty)$ holds. This completes the proof.

Remark 4.3. The tracking control using conventional adaptive fuzzy SMC with fuzzy switching control term [28-30] does not tackle the problem of attenuation of the effect of the fuzzy approximation error. We usually have $\dot{V} \leq S w-\eta S^{T} \operatorname{sign}(S)$. In this case, it can be excepted that $S w$ should be very small if not equal to zero in [28-30] but here we get $\dot{V} \leq-\sum_{i=1}^{p} \eta_{i}\left|s_{i}\right|<0$, which improves the stability proof. In order to overcome this restriction, we have proposed $\widehat{\omega}$ to estimate the fuzzy approximation error $\omega$. 
Remark 4.4. In conventional adaptive fuzzy SMC design [24, 39, 40], there is often an assumption that the unknown external disturbance $D(t)$ is bounded by a known positive constant. However, it is often not possible to obtain the bound in complex situations. In order to overcome this restriction, the variable $D(t)$ is incorporated into the equivalent control term $u_{e q}$ and a fuzzy system is used to approximate $u_{e q}$. Therefore, knowledge of the bound of external disturbance is not necessary in the present paper.

\subsection{Direct Adaptive Fuzzy SMC with Variable Universe Fuzzy Switching Term (DAFSMC with VUFSW)}

As stated in Section 3.2, variable universe fuzzy system possesses high static precision by virtue of contraction-expansion factor. In this subsection, to accelerate response speed and improve the control accuracy, we consider an adaptive fuzzy SMC with VUFSW.

Similar to the analysis in Section 4.2, the variables $s_{i}$ and $k_{i}$ are still taken as the input and the output variables of the variable universe fuzzy system. Then the variable universe fuzzy switching control law $\widehat{k}_{i}$ of the $i$ th subsystem can be written as

$$
\widehat{k}_{i}=\beta_{i} \zeta_{s i}\left(s_{i}\right)^{T} \theta_{s i}
$$

where $\zeta_{s i}\left(s_{i}\right)=\left[A_{1}\left(s_{i} / \alpha_{i}\left(s_{i}\right)\right), \ldots, A_{i_{L}}\left(s_{i} / \alpha_{i}\left(s_{i}\right)\right)\right]^{T}$ and $\theta_{s i}=\left[b_{1}, b_{2}, \ldots, b_{i_{L}}\right]^{T}$.

Let $\beta^{*}=\operatorname{diag}\left(\beta_{1}^{*}, \beta_{2}^{*}, \ldots, \beta_{p}^{*}\right)$ be the optimal parameter vector, where

$$
\beta_{i}^{*}=\underset{\left|\beta_{i}\right| \leq M_{i}}{\arg \min }\left\{\sup _{s_{i} \in D_{s i}}\left|k_{i}-\beta_{i} \zeta_{s i}\left(s_{i}\right)^{T} \theta_{s i}\right|\right\}
$$

in which $M_{i}$ is the design constant. Similarly, since the ideal parameter $\beta_{i}^{*}$ is unknown, $\beta_{i}$ is employed to estimate $\beta_{i}^{*}$. Therefore, the overall control effort can be modified as

$$
u_{i}=\widehat{u}_{e q i}+\widehat{k}_{i}+u_{c i}=\xi_{e i}^{T}(x) \theta_{e i}+\beta_{i} \zeta_{s i}^{T}\left(s_{i}\right) \theta_{s i}+\widehat{\omega}_{i}
$$

or

$$
u=\widehat{u}_{e q}+\widehat{K}+u_{c}=\Xi_{e}^{T}(x) \Theta_{e}+\beta \Xi_{s}^{T}(S) \Theta_{s}+\widehat{\omega},
$$

where $\Xi_{s}(S)=\left[\operatorname{diag}\left(\zeta_{s 1}^{T}(s 1), \zeta_{s 2}^{T}(s 2), \ldots, \zeta_{s p}^{T}(s p)\right)\right]^{T}$ and $\Theta_{s}=\left[\theta_{s 1}^{T}, \theta_{s 2}^{T}, \ldots, \theta_{s p}^{T}\right]^{T}$.

Substituting (4.25) into (4.1), we obtain

$$
\begin{aligned}
G_{1}(x) \dot{S} & =G_{1}(x) v-F_{1}(x)-\left(\Xi_{e}^{T} \Theta_{e}+\beta \Xi_{s}^{T}(S) \Theta_{s}+\widehat{\omega}\right)-G_{1}(x) D(t) \\
& =(\omega-\widehat{\omega})-\left(\Xi_{e}^{T} \Theta_{e}-\Xi_{e}^{T} \Theta_{e}^{*}\right)-\left(\beta \Xi_{s}^{T}(S) \Theta_{s}-\beta^{*} \Xi_{s}^{T}(S) \Theta_{s}\right)-\beta^{*} \Xi_{s}^{T}(S) \Theta_{s}-\delta(x) I_{p} S .
\end{aligned}
$$


Let $\widetilde{\Theta}_{e}=\Theta_{e}-\Theta_{e}^{*}$ and $\tilde{\beta}=\beta-\beta^{*}$. Then

$$
G_{1}(x) \dot{S}=\tilde{\omega}-\Xi_{e}^{T}(x) \tilde{\Theta}_{e}-\tilde{\beta} \Xi_{s}^{T}(S) \Theta_{s}-\beta^{*} \Xi_{s}^{T}(S) \Theta_{s}-\delta(x) I_{p} S .
$$

To derive the adaptive law of the parameter vectors, we consider the Lyapunov candidate function as

$$
V_{2}=\frac{1}{2} S^{T} G_{1}(x) S+\frac{1}{2 \gamma_{1}} \tilde{\Theta}_{e}^{T} \widetilde{\Theta}_{e}+\frac{1}{2 \gamma_{3}} \tilde{\beta}^{T} \tilde{\beta}^{T}+\frac{1}{2 \gamma} \tilde{\omega}^{T} \tilde{\omega},
$$

where $\gamma_{3}$ is a positive constant.

Theorem 4.5. For system (2.1), nonlinear function matrices $F(x), G(x)$, and vector $D(t)$ are unknown, and Assumptions 2.1-2.3 hold. The control law is selected as (4.24) or (4.25), with adaptation laws (4.29) as follows:

$$
\begin{gathered}
\dot{\theta}_{e i}= \begin{cases}\gamma_{1} s_{i} \xi_{e i}(x), & \left\|\theta_{e i}\right\|<M_{e i} \text { or }\left\|\theta_{e i}\right\|=M_{e i}, \gamma_{1} s_{i} \theta_{e i}^{T} \xi_{e i}(x) \leq 0, \\
\gamma_{1} s_{i} \xi_{e i}(x)-\gamma_{1} s_{i} \frac{\theta_{e i} \theta_{e i}^{T}}{\left\|\theta_{e i}\right\|^{2}} \xi_{e i}(x), & \left\|\theta_{e i}\right\|=M_{e i}, \gamma_{1} s_{i} \theta_{e i}^{T} \xi_{e i}(x)>0,\end{cases} \\
\dot{\beta}_{i}= \begin{cases}\gamma_{3} s_{i} \theta_{s i}^{T} \zeta_{s i}\left(s_{i}\right), & \left|\beta_{i}\right|<M_{i} \text { or }\left|\beta_{i}\right|=M_{i}, \gamma_{3} s_{i} \theta_{s i}^{T} \zeta_{s i}\left(s_{i}\right) \leq 0, \\
0, & \left|\beta_{i}\right|=M_{i}, \gamma_{3} s_{i} \theta_{s i}^{T} \zeta_{s i}\left(s_{i}\right)>0, \\
\dot{\hat{\omega}}=\gamma s_{i},\end{cases}
\end{gathered}
$$

where $M_{e i}$ and $M_{i}$ are defined as before. Then one can derive the performance as follows.

(1) The involved signals of the close loop are bounded.

(2) The tracking errors and their derivatives decay to zero asymptotically, in other words, when $t \rightarrow \infty, e_{i}^{(j)} \rightarrow 0, j=0,1, \ldots, r_{i}-1, i=1,2, \ldots, p$.

Proof. Differentiating (4.28) with respect to $t$ and using (4.27), we have

$$
\begin{aligned}
\dot{V}_{2}= & S^{T} G_{1}(x) \dot{S}+\frac{1}{2} S^{T} \dot{G}_{1}(x) S+\frac{1}{\gamma_{1}} \widetilde{\Theta}_{e}^{T} \dot{\tilde{\Theta}}_{e}+\frac{1}{\gamma_{3}} \tilde{\beta}^{T} \dot{\tilde{\beta}}+\frac{1}{\gamma} \tilde{\omega}^{T} \dot{\tilde{\omega}} \\
= & S^{T}\left(\tilde{\omega}-\Xi_{e}^{T}(x) \widetilde{\Theta}_{e}-\tilde{\beta}^{T} \Xi_{s}^{T}(S) \Theta_{s}-\beta^{*} \Xi_{s}^{T}(S) \Theta_{s}-\delta(x) I_{p} S\right) \\
& +\frac{1}{2} S^{T} \dot{G}_{1}(x) S+\frac{1}{\gamma_{1}} \widetilde{\Theta}_{e}^{T} \dot{\tilde{\Theta}}_{e}+\frac{1}{\gamma_{3}} \tilde{\beta}^{T} \dot{\tilde{\beta}}+\frac{1}{\gamma} \tilde{\omega}^{T} \dot{\tilde{\omega}}
\end{aligned}
$$




$$
\begin{aligned}
\leq & \sum_{i=1}^{p} s_{i} \tilde{\omega}_{i}-\frac{1}{\gamma} \sum_{i=1}^{p} \tilde{\omega}_{i}^{T} \dot{\hat{\omega}}_{i}-\sum_{i=1}^{p} s_{i} \tilde{\theta}_{e i}^{T} \xi_{e i}(x)+\frac{1}{\gamma_{1}} \sum_{i=1}^{p} \tilde{\theta}_{e i}^{T} \dot{\theta}_{e i}+\frac{1}{2} S^{T} \dot{G}_{1}(x) S-\delta(x)\|S\|^{2} \\
& -\sum_{i=1}^{p} s_{i} \tilde{\beta}_{i} \theta_{s i}^{T} \zeta_{s i}\left(s_{i}\right)+\frac{1}{\gamma_{3}} \sum_{i=1}^{p} \tilde{\beta}_{i} \dot{\beta}_{i}-\sum_{i=1}^{p} \eta_{i}\left|s_{i}\right| \\
\leq & \frac{1}{\gamma} \sum_{i=1}^{p} \tilde{\omega}_{i}\left(\gamma s_{i}-\dot{\hat{\omega}}_{i}\right)-\frac{1}{\gamma_{1}} \sum_{i=1}^{p} \tilde{\theta}_{e i}^{T}\left(\gamma_{1} s_{i} \xi_{e i}(x)-\dot{\theta}_{e i}\right)-\frac{1}{\gamma_{3}} \sum_{i=1}^{p} \tilde{\beta}_{i}\left(\gamma_{3} s_{i} \theta_{s i}^{T} \zeta_{s i}\left(s_{i}\right)-\dot{\beta}_{i}\right)-\sum_{i=1}^{p} \eta_{i}\left|s_{i}\right| .
\end{aligned}
$$

Noticing that (4.29), we have

$$
\dot{V}_{2} \leq-\sum_{i=1}^{p} \eta_{i}\left|s_{i}\right|<0
$$

Therefore, all signals in the system are bounded. In order to show the boundedness of $\dot{S}$, we rearrange (4.27) as follows:

$$
\dot{S}=G(x)\left(\tilde{\omega}-\Xi_{e}^{T}(x) \tilde{\Theta}_{e}-\tilde{\beta} \Xi_{s}^{T}(S) \Theta_{s}-\beta^{*} \Xi_{s}^{T}(S) \Theta_{s}-\delta(x) I_{p} S\right)
$$

Similarly, we can derive $\dot{S} \in L_{\infty}$.

To summarize the above analysis, the step-by-step procedures for the two direct adaptive fuzzy SMCs are proposed as follows.

Design Procedure:

Step 1. Select proper positive coefficients $\lambda_{1}, \lambda_{2}, \ldots, \lambda_{p}$ and learning coefficients $\gamma_{1}, \gamma_{2}, \gamma_{3}$, and $\gamma$.

Step 2. Specify design constant vectors $M_{e}=\left[M_{e 1}, M_{e 2}, \ldots, M_{e p}\right]^{T}, M_{s}=\left[M_{s 1}, M_{s 2}, \ldots\right.$, $\left.M_{s p}\right]^{T}$ and $M=\left[M_{1}, M_{2}, \ldots, M_{p}\right]^{T}$.

Step 3. Define $m_{i}$ fuzzy sets $F_{i}$ for variable $x_{i}$ to achieve an uniform coverage of the universe of discourse. Select the initial parameter vector $\theta_{e i}(0)=\underline{0}_{m_{i} \times 1}(i=1,2, \ldots, p)$.

Step 4. Construct the fuzzy rule bases for the fuzzy system $u_{\text {swi }}$. Define $i_{L}$ fuzzy sets $A_{i}$ for variable $s_{i}$ to achieve a uniform coverage of the universe of discourse. Select the initial parameter vectors $\theta_{s i}(0)_{i_{L} \times 1}(i=1,2, \ldots, p)$ for DAFSMC with FSW and the initial values $\beta_{i}(0)(i=1,2, \ldots, p)$ for DAFSMC with VUFSW.

Step 5. Construct the fuzzy systems $\widehat{u}_{e q i}$ in (4.9), and $\widehat{k}_{i}$ in (4.11) or (4.22).

Step 6. Construct the control law (4.13) or (4.14) with the adaptive laws in (4.18) or construct the control law (4.24) or (4.25) with the adaptive laws in (4.29).

Step 7. Use the adaptive laws (4.18) or (4.29) to adjust the parameters $\theta_{e i}, \theta_{s i}$ or $\beta_{i}$, and $\widehat{\omega}_{i}$. 


\section{Simulation Results and Analysis}

In this section, we test the proposed control schemes on the trajectory tracking control of the two-link rigid robot manipulators moving a horizontal plane. The equations of motion of the manipulators can be expressed in matrix form as follows [21, 25]:

$$
\left(\begin{array}{l}
\ddot{q}_{1} \\
\ddot{q}_{2}
\end{array}\right)=-\left(\begin{array}{ll}
M_{11}, & M_{12} \\
M_{21}, & M_{22}
\end{array}\right)^{-1}\left(\begin{array}{cc}
-h \dot{q}_{2}, & -h\left(\dot{q}_{1}+\dot{q}_{2}\right) \\
h \dot{q}_{1}, & 0
\end{array}\right)\left(\begin{array}{l}
\dot{q}_{1} \\
\dot{q}_{2}
\end{array}\right)+\left(\begin{array}{ll}
M_{11}, & M_{12} \\
M_{21}, & M_{22}
\end{array}\right)^{-1}\left(\begin{array}{l}
u_{1} \\
u_{2}
\end{array}\right)+\left(\begin{array}{l}
d_{1} \\
d_{2}
\end{array}\right),
$$

where

$$
\begin{gathered}
M_{11}=a_{1}+2 a_{3} \cos q_{2}+2 a_{4} \sin q_{2}, \\
M_{22}=a_{2}, \\
M_{21}=M_{12}=a_{2}+a_{3} \cos q_{2}+a_{4} \sin q_{2}, \\
h=a_{3} \sin q_{2}-a_{4} \cos q_{2},
\end{gathered}
$$

in which

$$
\begin{gathered}
a_{1}=I_{1}+m_{1} l_{c 1}^{2}+I_{e}+m_{e} l_{c e}^{2}+m_{e} l_{1}^{2}, \\
a_{2}=I_{e}+m_{e} l_{c e}^{2} \\
a_{3}=m_{e} l_{1} l_{c e} \cos \delta_{e} \\
a_{4}=m_{e} l_{1} l_{c e} \sin \delta_{e} .
\end{gathered}
$$

In the simulation, the following parameter values of the plant are used: $m_{1}=1, m_{e}=2$, $l_{1}=1, l_{c 1}=0.5, l_{c e}=0.6, I_{1}=0.12, I_{e}=0.25, \delta_{e}=30^{\circ}$. Let $y=\left[q_{1}, q_{2}\right]^{T}, u=\left[u_{1}, u_{2}\right]^{T}$, $x=\left[q_{1}, \dot{q}_{1}, q_{2}, \dot{q}_{2}\right]^{T}$,

$$
\begin{gathered}
F(x)=\left(\begin{array}{l}
f_{1}(x) \\
f_{2}(x)
\end{array}\right)=-\left(\begin{array}{ll}
M_{11}, & M_{12} \\
M_{21}, & M_{22}
\end{array}\right)^{-1}\left(\begin{array}{cc}
-h \dot{q}_{2}, & -h\left(\dot{q}_{1}+\dot{q}_{2}\right) \\
h \dot{q}_{1}, & 0
\end{array}\right)\left(\begin{array}{l}
\dot{q}_{1} \\
\dot{q}_{2}
\end{array}\right), \\
G(x)=\left(\begin{array}{ll}
g_{11}, & g_{12} \\
g_{21}, & g_{22}
\end{array}\right)=\left(\begin{array}{ll}
M_{11}, & M_{12} \\
M_{21}, & M_{22}
\end{array}\right)^{-1}, \quad D(t)=\left(\begin{array}{l}
d_{1} \\
d_{2}
\end{array}\right) .
\end{gathered}
$$

Then, the robotic manipulators dynamics given by (5.1) can be expressed as

$$
\ddot{y}=F(x)+G(x) u+D(t) .
$$

The object is to design control law $u$ to force the system output $q_{1}$ and $q_{2}$ to track the desired trajectories $y_{d 1}=\sin t$ and $y_{d 2}=\sin t$, respectively. The initial state is selected as $x_{0}=[1,0,1,0]^{T}$. The fuzzy system $\widehat{k}_{i}(i=1,2)$ in (4.9) have $x=\left[q_{1}, \dot{q}_{1}, q_{2}, \dot{q}_{2}\right]^{T}$ as inputs. For each input variable, we define three triangular membership functions uniformly distributed on the interval $[-1,1] . s_{i}$ and $k_{i}$ are the input and the output of the fuzzy system $\widehat{u}_{\text {swi }}(i=1,2)$ 
Table 1: Fuzzy rule list of variable universe fuzzy switching control.

\begin{tabular}{llllllll}
\hline Input $s_{i}$ & PB & PM & PS & ZE & NS & NM & NB \\
Output $k_{i}$ & PB & PM & PS & ZE & NS & NM & NB \\
\hline
\end{tabular}

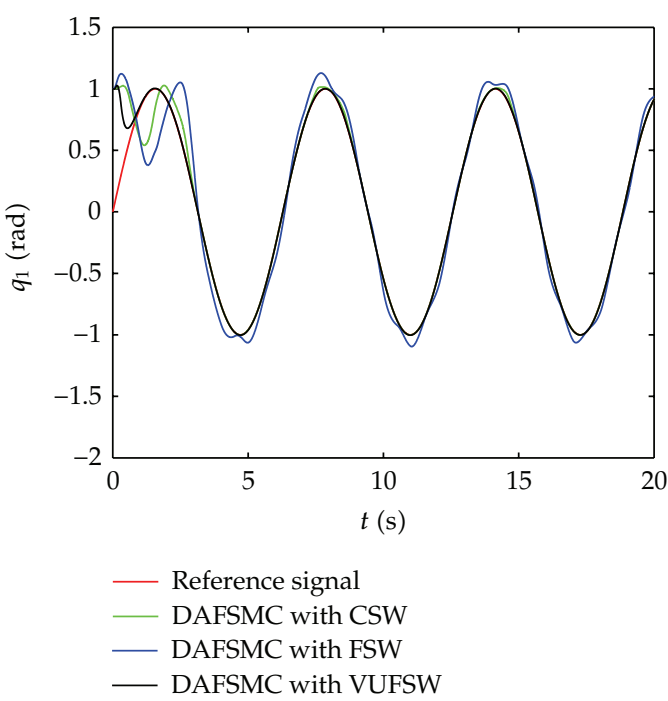

(a) Tracking curve of $q_{1}$

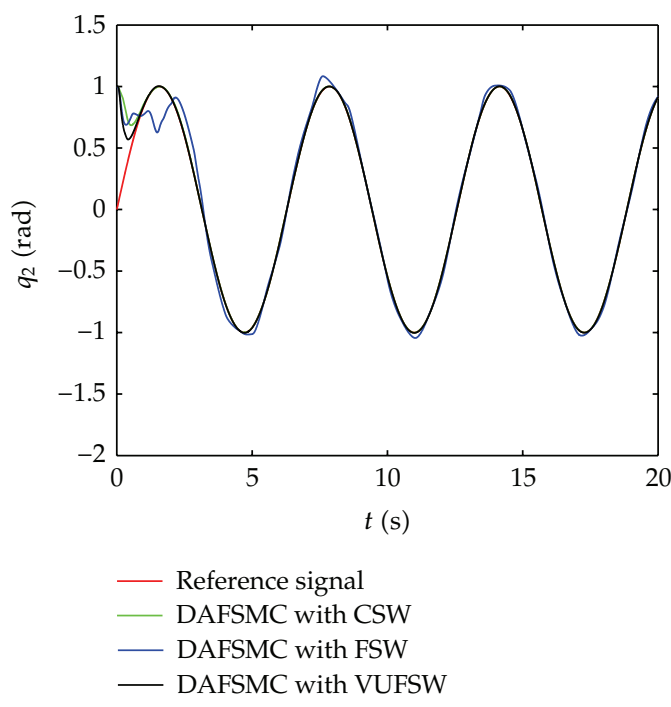

(b) Tracking curve of $q_{2}$

Figure 1: Angular trajectory tracking curves of robotic manipulators.

in (4.11) and (4.22), respectively. We define seven triangle membership functions uniformly distributed on the input domain $[-2,2]$ and the output domain $[-1,1]$, respectively. The used fuzzy rules are showed in Table 1, in which the fuzzy labels used in this study are negative big (NB), negative medium (NM), negative small (NS), zero (ZE), positive small (PS), positive medium (PM), and positive big (PB).

In this paper, we consider that the nonlinear function matrices $F(x)$ and $G(x)$ are assumed to be completely unknown, that is, the design of the proposed controller does not require the knowledge of the system's model. Moreover, the external disturbance $D(t)$ is also unknown. In fact, these functions are only required for simulation purpose. To make a fair comparison, in the simulation, we consider the direct adaptive fuzzy SMC proposed by $A$. Boulkroune et al. in [21] where a classical switching control term is employed to eliminate the fuzzy approximation error (hereafter referred to as a DAFSMC with CSW).

In the whole simulation, the design parameters used are chosen as follows: $\lambda_{1}=\lambda_{2}=5$, $\gamma_{1}=1, \gamma_{2}=8, \gamma_{3}=8, \gamma=1, M_{e 1}=2, M_{e 2}=1, M_{s 1}=50, M_{s 2}=20, M_{1}=M_{2}=2$, $\eta_{1}=13, \eta_{2}=6$ and the contraction-expansion factors $\alpha_{i}=1-\lambda \exp \left(-k s_{i}^{2}\right)(i=1,2)$ with $\lambda=0.95, k=1$. The initial conditions of the online adjustable parameter vectors are selected as $\theta_{e i}=[0]_{81 \times 1}(i=1,2), \theta_{s i}=[-3,-2,-1,0,1,2,3]^{T}(i=1,2)$, and $\beta(0)=\operatorname{diag}(5,1)$.

To verify the robust stability of the proposed schemes, external disturbances are chosen as square wave signal $d_{1}=d_{2}=$ square $(2 \pi t)$ [21]. The response curves, the tracking error curves, and sliding mode dynamic evolution curves under the aforementioned three control schemes are illustrated in Figures 1, 2, and 3, respectively. Their associated control efforts are illustrated in Figure 4. For evaluating numerically their tracking performance, the integral 


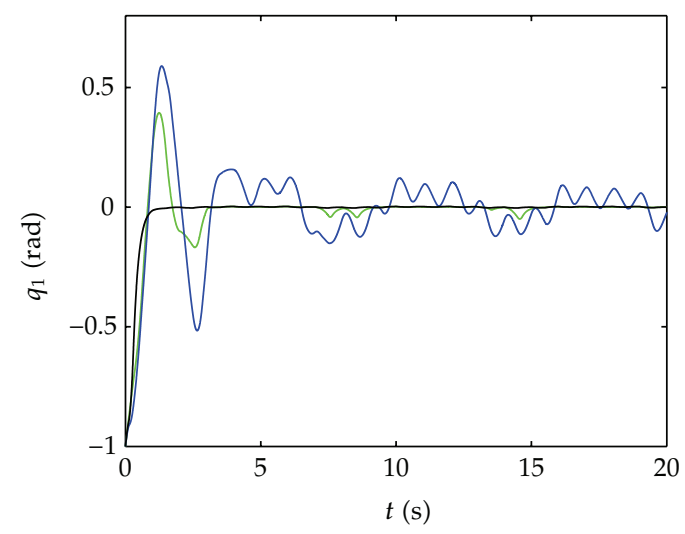

- DAFSMC with CSW

— DAFSMC with FSW

— DAFSMC with VUFSW

(a) Tracking error of $q_{1}$

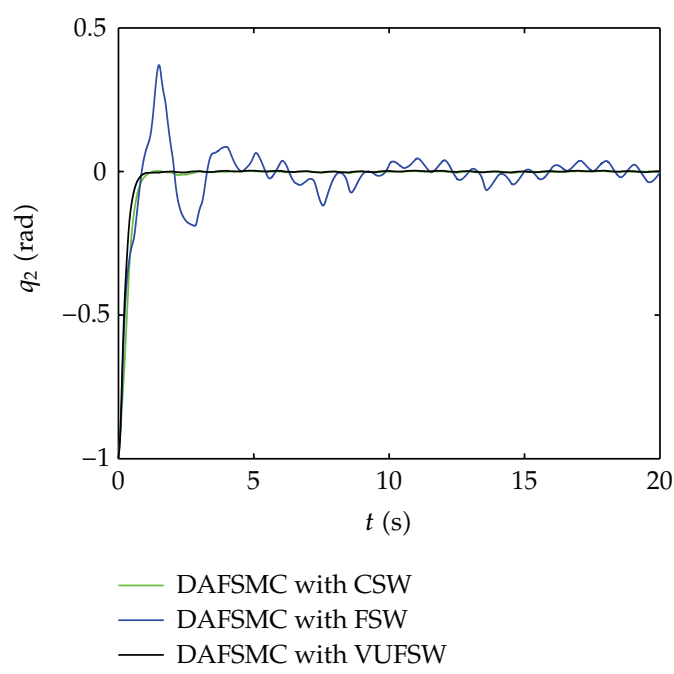

(b) Tracking error of $q_{2}$

Figure 2: Angular trajectory tracking error curves of robotic manipulators.

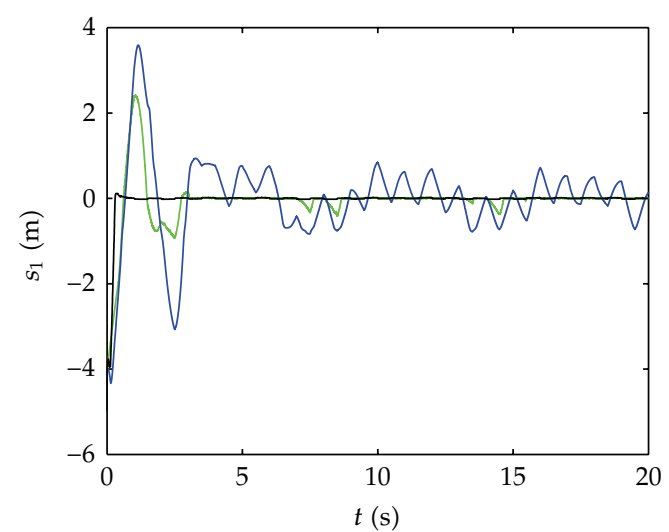

— DAFSMC with CSW

— DAFSMC with FSW

- DAFSMC with VUFSW

(a) Sliding surface $s_{1}$

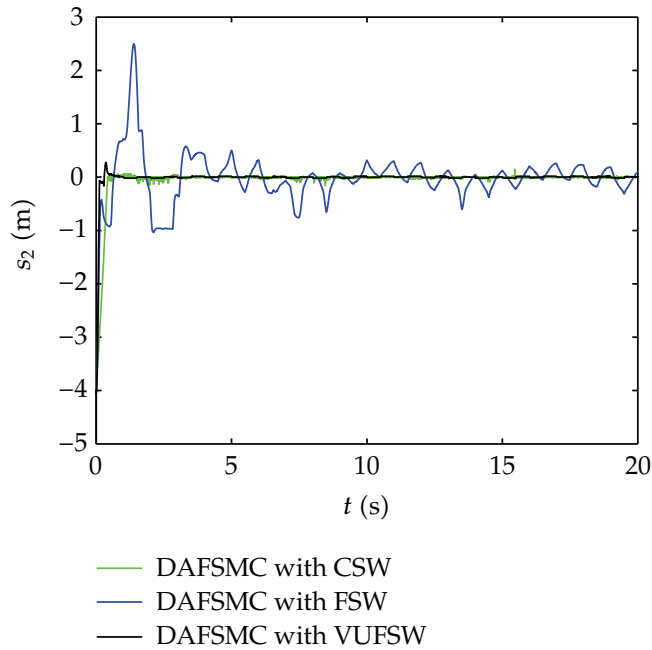

(b) Sliding surface $s_{2}$

Figure 3: Sliding mode dynamic evolution curves.

of the absolute error (IAE), the integral of the time multiplied by the absolute error (ITAE), and the integral of square value (ISV) of the control input are also considered because mere visual observation of response curve is not always enough to make a sound comparison. The corresponding tracking performance indices in first 20 seconds and in first 100 seconds are tabulated in Tables 2 and 3, respectively.

As shown in Figures 1-4, the proposed two schemes can effectively achieve the trajectory tracking of the joint angles despite of the system uncertainties and external disturbances. Furthermore, they can effectively alleviate the chattering, which is the main 


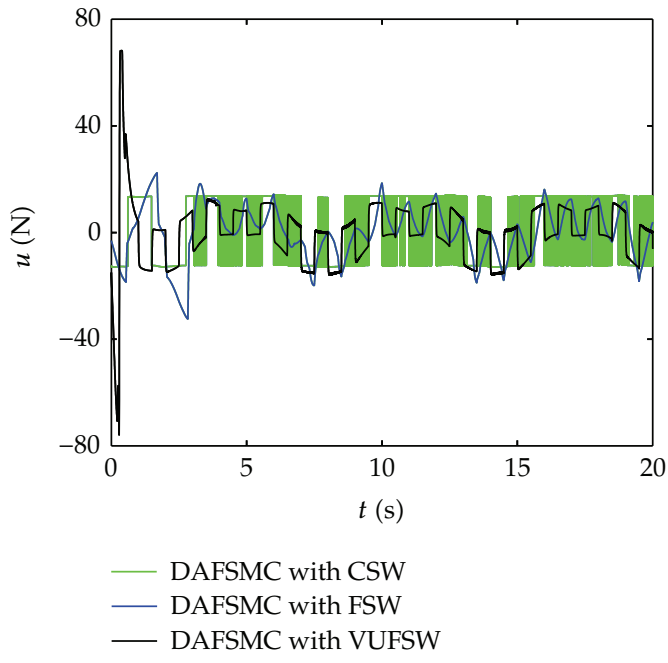

(a) Control effort of $q_{1}$

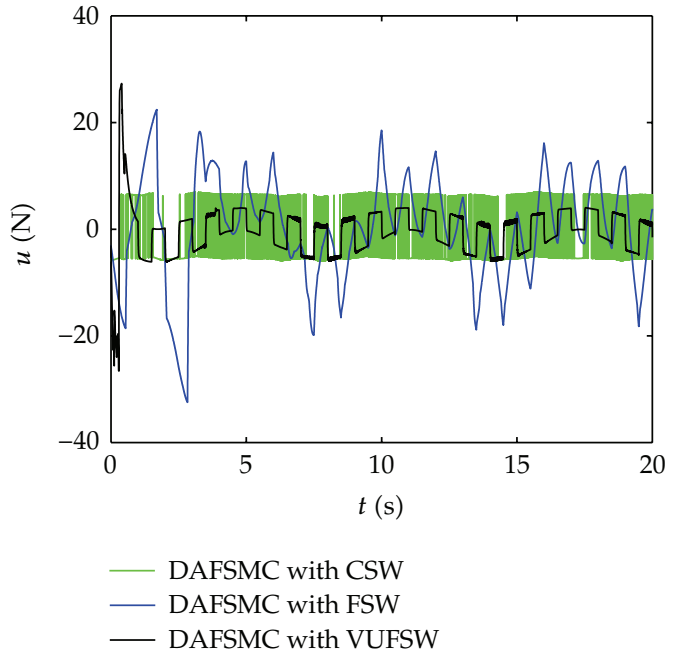

(b) Control effort of $q_{2}$

Figure 4: Control input torques of robotic manipulators.

Table 2: The performance indices in 20 seconds.

\begin{tabular}{lcccccc}
\hline Controller & \multicolumn{3}{c}{ rod1 } & \multicolumn{3}{c}{ rod2 } \\
& IAE $(\mathrm{rad})$ & ITAE $(\mathrm{rad} \cdot \mathrm{s})$ & ISV $\left(\mathrm{N}^{2}\right)$ & IAE $(\mathrm{rad})$ & ITAE $(\mathrm{rad} \cdot \mathrm{s})$ & ${\text { ISV }\left(\mathrm{N}^{2}\right)}^{\text {ITA }}$ \\
\hline DAFSMC with CSW & 0.8485 & 1.5820 & $3.3838 e+03$ & 0.4191 & 0.3299 & 704.3263 \\
DAFSMC with FSW & 2.3991 & 12.8742 & $1.9102 e+03$ & 1.1504 & 5.3901 & 253.8947 \\
DAFSMC with VUFSW & 0.3923 & 0.3867 & $2.9613 e+03$ & 0.2852 & 0.3180 & 437.4319 \\
\hline
\end{tabular}

disadvantage of general SMC. It can also be seen from Figures 1-3, the performance specifications of DAFSMC with VUFSW are much better than those of DAFSMC with CSW and DAFSMC with FSW in terms of reaching time, steady precision.

Both IAE and ITAE are used as evaluating error performance, while the criterion ISV shows energy consumption. It is well known that there is a trade-off between error performance and energy consumption, that is, when IAE and ITAE are improved, ISV becomes worse, and vice versa. Conservative control input is often required to guarantee the stability of the control system in DAFSMC with CSW scheme. Therefore, DAFSMC with CSW expends relatively more energy to achieve the tracking task than DAFSMC with FSW. It also implies that the indices IAE and ITAE of DAFSMC with FSW become worse. But DAFSMC with VUFSW simultaneously improves IAE and ITAE as well as ISV as compared to the others as stated in Tables 2 and 3. It is undeniable that DAFSMC with VUFSW expends more energy than DAFSMC with FSW in the initial stage due to its fast response speed. However, thanks to the high precision of the variable universe fuzzy control, its energy consumption reduces quickly in the steady state.

Remark 5.1. As already stated in Section 1, in the presence of model uncertainties and external disturbances, the two disadvantages of general SMC cannot be overcome just using fuzzy switching technique or adaptive fuzzy SMC. Combining adaptive fuzzy SMC with fuzzy or variable universe fuzzy switching technique, a novel framework for designing direct 
Table 3: The performance indices in 100 seconds.

\begin{tabular}{lcccccc}
\hline \multirow{2}{*}{ Controller } & \multicolumn{3}{c}{ rod1 } & \multicolumn{3}{c}{ rod2 } \\
& IAE $(\mathrm{rad})$ & ITAE $(\mathrm{rad} \cdot \mathrm{s})$ & ISV $\left(\mathrm{N}^{2}\right)$ & IAE $(\mathrm{rad})$ & ITAE $(\mathrm{rad} \cdot \mathrm{s})$ & $\mathrm{ISV}_{\left(\mathrm{N}^{2}\right)}$ \\
\hline DAFSMC with CSW & 1.4397 & 33.2521 & $1.6863 e+04$ & 0.4722 & 6.6922 & $3.5550 e+03$ \\
DAFSMC with FSW & 3.9996 & 97.6183 & $9.0121 e+03$ & 2.1688 & 63.2731 & $1.2830 e+03$ \\
DAFSMC with VUFSW & 0.5073 & 7.2268 & $8.6772 e+03$ & 0.3844 & 6.1505 & $1.2590 e+03$ \\
\hline
\end{tabular}

adaptive fuzzy SMC is developed for a class of MIMO nonlinear systems in this study. The derived schemes can effectively overcome these disadvantages of the general SMC.

Remark 5.2. Compared with [22, 24, 28-30,38, 41], our proposed control schemes have several advantages as follows. (i) The proposed schemes can simultaneously solve the chattering and the calculation of the equivalent control in the presence of model uncertainties and unknown disturbances. (ii) The constraint on the control gain matrix is relaxed. In this study, the gain matrix is just required to be positive definite symmetric and the inverse of its derivative is bounded by an unknown function. (iii) An adaptive compensation term is appended to remove the assumption on fuzzy approximation error in the stability proof. (iv) The derived DAFSMC with VUFSW achieves better performances than the others in terms of response speed, steady accuracy, IAE, ITAE, and ISV.

\section{Conclusion}

A novel framework is developed to design a direct adaptive fuzzy SMC for a class of MIMO nonlinear systems with model uncertainties and unknown disturbances. Combining adaptive fuzzy SMC with fuzzy or variable universe fuzzy switching technique, this study proposes two novel direct adaptive fuzzy SMC schemes. The derived schemes effectively overcome the two disadvantages of general SMC. Besides, the constraint on the control gain matrix and the fuzzy approximation error are relaxed. Future works will focus on the extension of the framework to more general MIMO nonlinear systems such as the continuous-time or the discrete-time nonaffine nonlinear systems.

\section{Acknowledgments}

This work was partly supported by NSFC project (no. 61074044), Basic Scientific and Technological Frontier project of Henan province (no. 092300410178), Scientific Research key project of Henan provincial Education Department (no. 12B110007), and Specialized Research Fund for the Doctoral Program of Higher Education (no. 20090041110003).

\section{References}

[1] H. Hu and P. Y. Woo, "Fuzzy supervisory sliding-mode and neural-network control for robotic manipulators," IEEE Transactions on Industrial Electronics, vol. 53, no. 3, pp. 929-940, 2006.

[2] Z. S. Song, J. Q. Yi, D. B. Zhao, and X. Li, "A computed torque controller for uncertain robotic manipulator systems: fuzzy approach," Fuzzy Sets and Systems, vol. 154, no. 2, pp. 208-226, 2005.

[3] J. J. Slotine and W. Li, Applied Nonlinear Control), Prentice-Hall, Englewood Cliffs, NJ, USA, 1991.

[4] L. G. Wu, X. J. Su, and P. Shi, "Sliding mode control with bounded $L_{2}$ gain performance of Markovian jump singular time-delay systems," Automatica, vol. 48, pp. 1929-1933, 2012. 
[5] J. T. Fei M and Y. Xin, "Robust adaptive sliding mode controller for semi-active vehicle suspension system," International Journal of Innovative Computing Information and Control, vol. 8, no. 1, pp. 691-700, 2012.

[6] Q. Khan, A. I. Bhatti, M. Iqbal et al., "Dynamic integral sliding mode control for SISO uncertain nonlinear systems," International Journal of Innovative Computing Information and Control, vol. 8, no. 7, pp. 4621-4633, 2012.

[7] M. Ertugrul and O. Kaynak, "Neuro sliding mode control of robotic manipulators," Mechatronics, vol. 10, pp. 243-267, 2000.

[8] X. J. Su, P. Shi, L. G. Wu et al., "A novel control design on discrete-timeTakagi-Sugeno fuzzy systems with timevarying delays," IEEE Transactions on Fuzzy Systems. In press.

[9] X. J. Su, P. Shi, L. G. Wu et al., "A novel approach to filter design for T-S fuzzy discrete-time systems with timevarying delay," IEEE Transactions on Fuzzy Systems, vol. 20, no. 6, pp. 1114-1129, 2012.

[10] L. X. Wang and J. M. Mendel, "Fuzzy basis functions, universal approximation, and orthogonal leastsquares learning," IEEE Transactions on Neural Networks, vol. 3, no. 5, pp. 807-814, 1992.

[11] L. G. Wu, X. J. Su, P. Shi et al., "Model approximation for discrete-time state-delay systems in the T-S fuzzy framework," IEEE Transactions on Fuzzy Systems, vol. 19, no. 2, pp. 366-378, 2011.

[12] Q. Zhou, P. Shi, S. Y. Xu et al., "Adaptive output feedbackcontrol for nonlinear time-delay systems by fuzzy approximation approach. IEEE," Transactions on Fuzzy Systems. In press.

[13] Q. Zhou, P. Shi, J. J. Lu et al., "Adaptive output-feedback fuzzy tracking control for a class of nonlinear systems," IEEE Transactions on Fuzzy Systems, vol. 19, no. 5, pp. 972-982, 2011.

[14] T. C. Lin, S. W. Chang, and C. H. Hsu, "Robust adaptive fuzzy sliding mode control for a class of uncertain discrete-time nonlinear systems," International Journal of Innovative Computing Information and Control, vol. 8, no. 1, pp. 347-359, 2012.

[15] M. Roopaei and M. Zolghadri Jahromi, "Chattering-free fuzzy sliding mode control in MIMO uncertain systems," Nonlinear Analysis. Theory, Methods \& Applications, vol. 71, no. 10, pp. 4430-4437, 2009.

[16] H. T. Yau and C. L. Chen, "Chattering-free fuzzy sliding-mode control strategy for uncertain chaotic systems," Chaos, Solitons and Fractals, vol. 30, no. 3, pp. 709-718, 2006.

[17] N. Sadati and R. Ghadami, "Adaptive multi-model sliding mode control of robotic manipulators using soft computing," Neurocomputing, vol. 71, pp. 2702-2710, 2008.

[18] H. Lee, E. Kim, H.-J. Kang, and M. Park, "A new sliding-mode control with fuzzy boundary layer," Fuzzy Sets and Systems, vol. 120, no. 1, pp. 135-143, 2001.

[19] Y. R. Hwang and M. Tomizuka, "Fuzzy smoothing algorithms for variable structure systems," IEEE Transactions on Fuzzy Systems, vol. 2, no. 4, pp. 277-284, 1994.

[20] M. H. Korayem, R. Haghighi, A. H. Korayem et al., "Determining maximum load carrying capacity of planar flexiblelink robot: closed-loop approach," Robotica, vol. 28, pp. 959-973, 2010.

[21] A. Boulkroune, M. Tadjine, M. M'Saad, and M. Farza, "Fuzzy adaptive controller for MIMO nonlinear systems with known and unknown control direction," Fuzzy Sets and Systems, vol. 161, no. 6, pp. 797820, 2010.

[22] J.-Y. Chen, "Rule regulation of fuzzy sliding mode controller design: direct adaptive approach," Fuzzy Sets and Systems, vol. 120, no. 1, pp. 159-168, 2001.

[23] Y. Byungkook and H. Woonchul, "Adaptive fuzzy sliding mode control of nonlinear system," IEEE Transactions on Fuzzy Systems, vol. 6, no. 2, pp. 315-321, 1998.

[24] H. F. Ho, Y. K. Wong, and A. B. Rad, "Robust fuzzy tracking control for robotic manipulators," Simulation Modelling Practice and Theory, vol. 15, no. 7, pp. 801-816, 2007.

[25] S. Labiod, M. S. Boucherit, and T. M. Guerra, "Adaptive fuzzy control of a class of MIMO nonlinear systems," Fuzzy Sets and Systems, vol. 151, no. 1, pp. 59-77, 2005.

[26] B. Yoo and W. Ham, "Adaptive fuzzy sliding mode control of nonlinear system," IEEE Transactions on Fuzzy Systems, vol. 6, no. 2, pp. 315-321, 1998.

[27] S. Frikha, M. Djemel, and N. Derbel, "Observer based adaptive neuro-sliding mode control for MIMO nonlinear systems," International Journal of Control, vol. 8, no. 2, pp. 257-265, 2010.

[28] P. T. Chan, A. B. Rad, and J. Wang, "Indirect adaptive fuzzy sliding mode control. II. Parameter projection and supervisory control," Fuzzy Sets and Systems, vol. 122, no. 1, pp. 31-43, 2001.

[29] J. Wang, A. B. Rad, and P. T. Chan, "Indirect adaptive fuzzy sliding mode control. I. Fuzzy switching," Fuzzy Sets and Systems, vol. 122, no. 1, pp. 21-30, 2001.

[30] M. Roopaei, M. Zolghadri, and S. Meshksar, "Enhanced adaptive fuzzy sliding mode control for uncertain nonlinear systems," Communications in Nonlinear Science and Numerical Simulation, vol. 14, no. 9-10, pp. 3670-3681, 2009. 
[31] P. A. Phan and T. J. Gale, "Direct Adaptive fuzzy control with less restrictions on the control gain," International Journal of Control Automation and Systems, vol. 5, no. 6, pp. 621-629, 2007.

[32] S. Labiod and T. M. Guerra, "Direct adaptive fuzzy control for a class of MIMO nonlinear systems," International Journal of Systems Science, vol. 38, no. 8, pp. 665-675, 2007.

[33] H.-X. Li, Z.-H. Miao, and E. S. Lee, "Variable universe stable adaptive fuzzy control of a nonlinear system," Computers E Mathematics with Applications, vol. 44, no. 5-6, pp. 799-815, 2002.

[34] S. Aloui, O. Pages, A. El Hajjaji et al., "Improved fuzzy sliding mode control for a class of MIMO nonlinear uncertain and perturbed systems," Applied Soft Computing, vol. 11, pp. 820-826, 2011.

[35] H. X. Li, "Interpolation mechanism of fuzzy control," Science in China E., vol. 41, no. 3, pp. 312-320, 1998.

[36] H. X. Li, "Adaptive fuzzy controllers based on variable universe," Science in China E, vol. 42, no. 1, pp. 10-20, 1999.

[37] H. X. Li, "Essence of fuzzy control in mathematics and design on a class of high-accuracy fuzzy controller," Control theory and Applications, vol. 14, no. 6, pp. 868-876, 1997.

[38] L. X. Wang, "Stable adaptive fuzzy control of nonlinear systems," IEEE Transactions on Fuzzy Systems, vol. 1, no. 2, pp. 146-155, 1993.

[39] M. R. Faieghi, H. Delavari, and D. Baleanu, "A novel adaptive controller for two-degree of freedom polar robot with unknown perturbations," Communications in Nonlinear Science and Numerical Simulation, vol. 17, no. 2, pp. 1021-1030, 2012.

[40] V. Nekoukar and A. Erfanian, "Adaptive fuzzy terminal sliding mode control for a class of MIMO uncertain nonlinear systems," Fuzzy Sets and Systems, vol. 179, pp. 34-49, 2011.

[41] Y. C. Chang, "Adaptive fuzzy-based tracking control for nonlinear SISO systems via VSS and approaches," IEEE Transactions on Fuzzy Systems, vol. 9, pp. 278-292, 2001. 


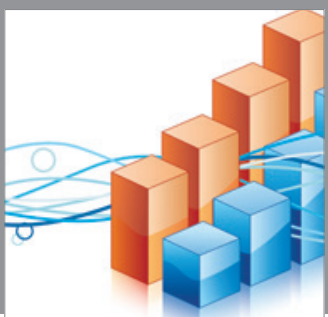

Advances in

Operations Research

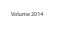

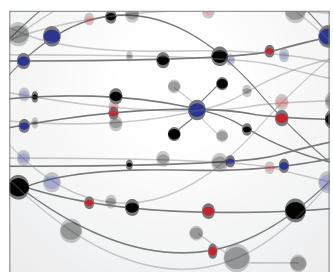

\section{The Scientific} World Journal
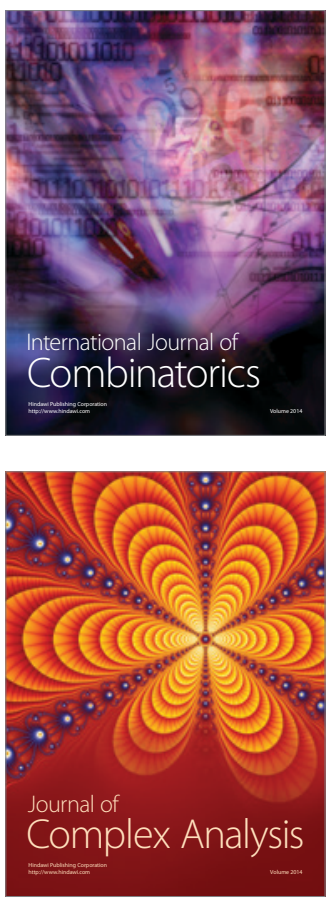

International Journal of

Mathematics and

Mathematical

Sciences
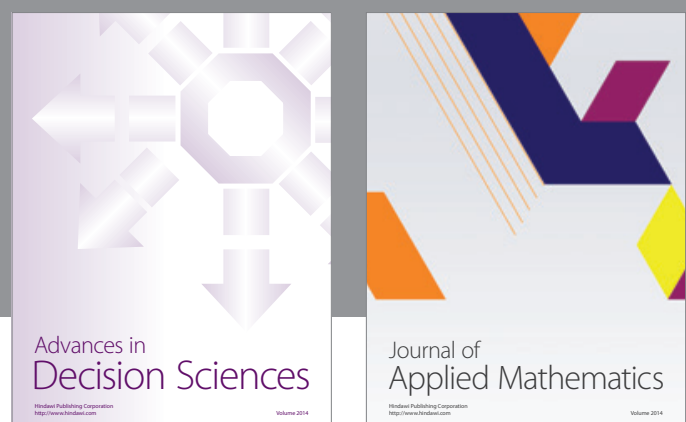

Journal of

Applied Mathematics
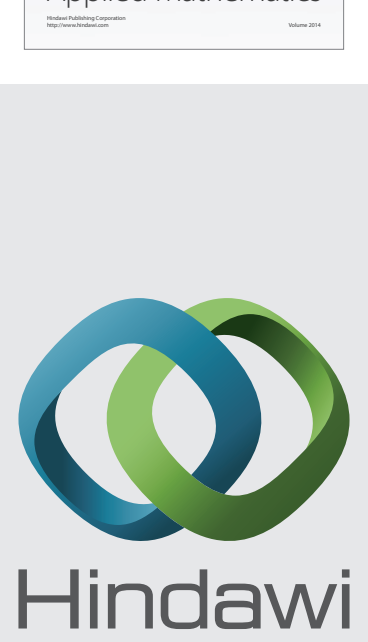

Submit your manuscripts at http://www.hindawi.com
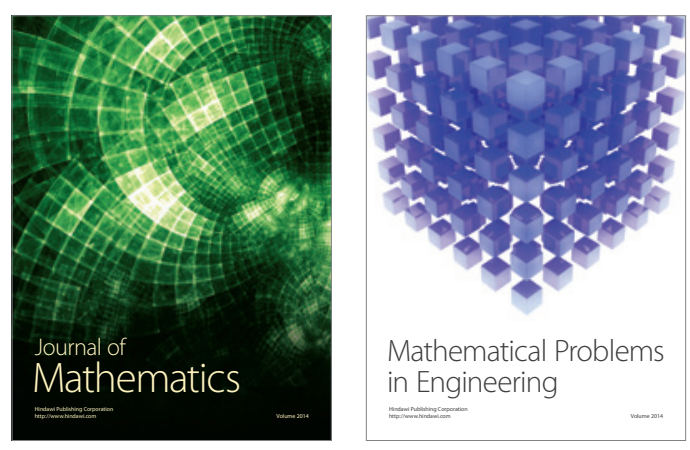

Mathematical Problems in Engineering
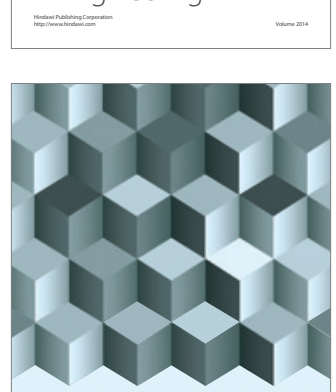

Journal of

Function Spaces
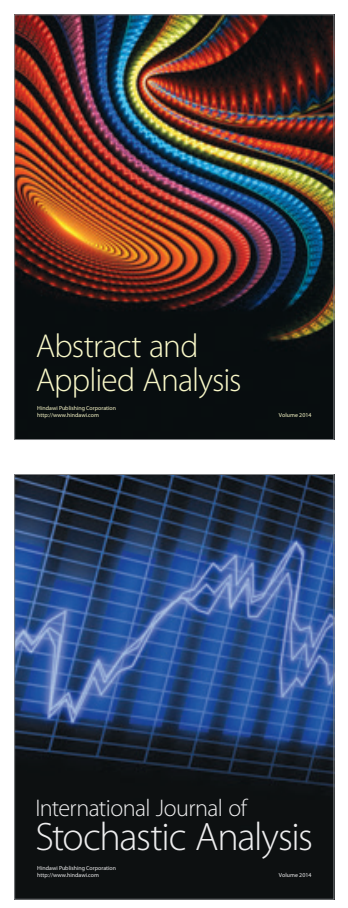

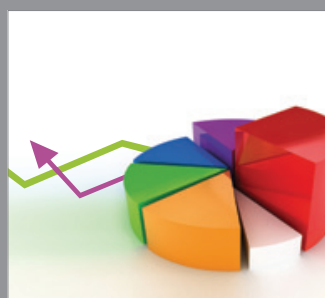

ournal of

Probability and Statistics

Promensencen
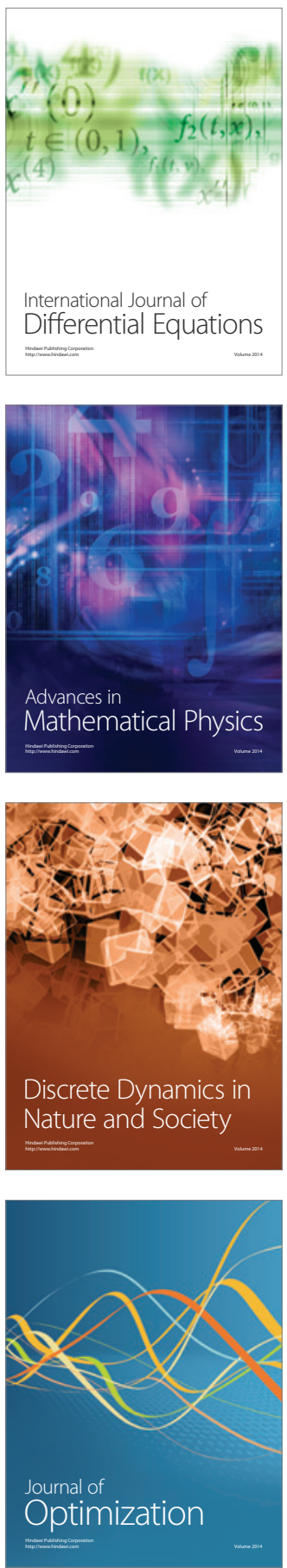